\title{
The weight of flash chromatography: A tool to predict its mass intensity from thin-layer chromatography
}

\author{
Freddy Pessel ${ }^{1}$, Jacques Augé ${ }^{2}$, Isabelle Billault ${ }^{1}$ and Marie-Christine Scherrmann ${ }^{* 1}$
}

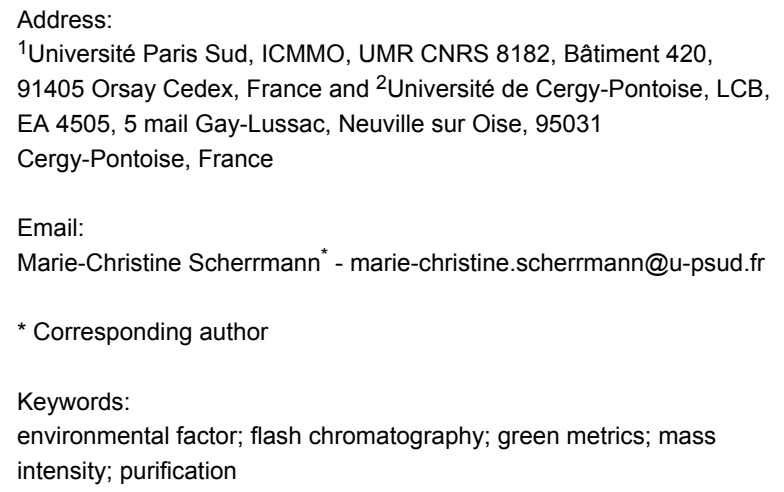

\begin{abstract}
Purification by flash chromatography strongly impacts the greenness of a process. Unfortunately, due to the lack of the relevant literature data, very often this impact cannot be assessed thus preventing the comparison of the environmental factors affecting the syntheses. We developed a simple mathematical approach to evaluate the minimum mass intensity of flash chromatography from the retention factor values determined by thin-layer chromatography.
\end{abstract}

\section{Introduction}

As part of a more respectful environmental chemistry, many efforts have been made to reduce the impact of chemical transformations by developing high atom-economic reactions, alternative reaction media or high-performance catalysts. The formation of a pure chemical product not only requires reactants, solvents, promoters and catalysts used in the reaction, but also other materials used for the work-up and for the purification steps. The Sheldon $E$ factor [1,2] and the mass intensity $M I$ [3-5], which are defined according to Equation 1 and Equation 2, respectively, are classical metrics based on the economy of material for evaluating the greenness of a process.

It is worth noting that these mass-based metrics allowed to quantify the mass of waste but did not take into account their potential for negative effects on the environment. These two metrics are related by Equation 3 [6].

$$
\begin{gathered}
E=\frac{\text { total mass of waste }}{\text { mass of product }} \\
M I=\frac{\text { total mass used in the process }}{\text { mass of product }} \\
M I=E+1
\end{gathered}
$$

The amount of waste includes the amount of the byproducts, but also the amount of non-reacting starting materials, auxiliaries, catalysts or any additives such as acids, bases, salts, solvents of 
the reaction or solvents required for the work-up and the purification. We demonstrated that the mass intensity could be easily calculated for linear and convergent sequences from the global material economy GME (Equation 4), which is related to the atom economy, the yields of each step, the excess of reactants and the mass of auxiliaries [6,7].

$$
M I=\frac{1}{G M E}
$$

It can be fractioned into three parts: reaction itself $\left(M I_{\mathrm{R}}\right)$, workup $\left(M I_{\mathrm{W}}\right)$ and purification $\left(M I_{\mathrm{P}}\right)$ as shown by Equation 5 [8].

$$
M I=M I_{\mathrm{R}}+M I_{\mathrm{W}}+M I_{\mathrm{P}}
$$

Any value of the $E$ factor which does not take into account the work-up and purification steps is nonsensical, since the values of $M I_{\mathrm{W}}$ and $M I_{\mathrm{P}}$ are often much higher than the value of $M I_{\mathrm{R}}$.

In order to compare the greenness of different processes, each term of Equation 5 has to be known. From the literature data it is possible to retrieve information concerning the amount of reactants, solvents and catalysts allowing the calculation of $M I_{\mathrm{R}}$. Moreover, since the work-up is usually well described, it is easy to gain access to $M I_{\mathrm{W}}$. In contrast, the amount of auxiliaries and solvents used in the purification of products is very often omitted. For example, the mass of silica gel and eluents used are never mentioned, which prevents the reader from calculating $M I_{\mathrm{p}}$, and thus having the actual value of the $E$ factor. The impact of chromatography on sustainability was recently discussed [9] and we propose here a method to evaluate such an item. This tool can also allow the chemist to evaluate, from a thin-layer chromatography (TLC), the minimum mass required to perform a flash chromatography. Our calculations are based on the preparative chromatographic technique largely used by chemists [10-12] and on our own experiments.

\section{Results and Discussion}

The publication of Still et al. [10] describing flash chromatography in 1978 greatly facilitated the post synthesis purifications which were, until then, often carried out by gravity column chromatography that was time consuming and did not always lead to effective separations. Since then, various automated systems equipped with pumps and eventually detectors and using disposable pre-packed silica cartridges were marketed offering great ease of use.

The mass intensity of purification by chromatography $\left(M I_{\mathrm{Chr}}\right)$ is the ratio between the total mass used to perform the chromatography (i.e., the sum of the mass of silica $\left(m_{\mathrm{SiO}_{2}}\right)$ and the mass of eluent ( $\left.\left.m_{\text {eluent }}\right)\right)$ and $m_{\mathrm{p}}$, the mass of the product (Equation 6 ).

$M I_{C h r}=\frac{\text { total mass used for chromatography }}{\text { mass of product }}=\frac{m_{\mathrm{SiO}_{2}}+m_{\mathrm{eluent}}}{m_{\mathrm{p}}}$

\section{Mass of silica}

The size of the column for chromatography and therefore the amount of silica and solvent depends on the mass of the sample and on the difficulty of separation of the products. This difficulty may be evaluated by $\Delta R_{\mathrm{f}}$ that is the difference between the retention factor $R_{\mathrm{f}}$ of products in TLC (thin-layer chromatography). Based on their experimentations, Still et al. recommended typical column diameters (constant height) and sample loading for difficult separations $\left(0.2>\Delta R_{\mathrm{f}} \geq 0.1\right)$ or more easier separations $\left(\Delta R_{\mathrm{f}} \geq 0.2\right)[10]$. Using a column height of 5.9 inches (ca. $15 \mathrm{~cm}$ ) and considering that the silica has a density of 0.5 , correlations have been established between the mass of silica to

\begin{tabular}{|c|c|c|c|c|c|c|}
\hline \multirow{2}{*}{ Entry } & \multirow{2}{*}{ Cartridge } & \multirow{2}{*}{ Particles shape } & \multirow{2}{*}{ Average particle size $(\mu \mathrm{m})$} & \multicolumn{3}{|c|}{$m_{\mathrm{SiO}_{2}}=f\left(m_{\mathrm{s}}\right)$} \\
\hline & & & & $\begin{array}{l}\text { difficult } \\
\text { separation }\end{array}$ & $\begin{array}{c}\text { moderately } \\
\text { difficult separation }\end{array}$ & $\begin{array}{c}\text { easy } \\
\text { separation }\end{array}$ \\
\hline 1 & Silica gel ${ }^{a}$ & irregular & $40-63$ & $151.2 m_{\mathrm{s}}+0.5$ & $59.8 m_{\mathrm{s}}$ & \\
\hline 2 & RediSep ${ }^{T M}$ & irregular & $35-70$ & $1000 m_{\mathrm{s}}$ & $25 m_{\mathrm{s}}$ & 14. $m_{\mathrm{s}}$ \\
\hline 3 & $\begin{array}{l}\text { EasyVario } \\
\text { Flash }^{T M}\end{array}$ & irregular & $15-40$ & & $33.3 m_{\mathrm{s}}$ & \\
\hline 4 & SNAPTM & irregular & $40-50$ & $10 m_{\mathrm{s}}$ & $20 m_{\mathrm{s}}$ & $10 m_{\mathrm{s}}$ \\
\hline 5 & SNAP Ultra ${ }^{\mathrm{TM}}$ & spherical & 25 & $50 m_{\mathrm{s}}$ & $10 m_{\mathrm{s}}$ & $5 m_{\mathrm{s}}$ \\
\hline
\end{tabular}
be used and mass $\left(m_{\mathrm{S}}\right)$ of the sample to be purified (Table 1,

aManually packed glass column. 
entry 1) [12]. For commercial pre-packed cartridge indications are also provided [13-15] and we have selected some data to obtain a general trend (Table 1).

The mass of silica required to purify $m_{\mathrm{s}} \mathrm{g}$ of sample may therefore be estimated by Equation 7. Excluding the equation obtained for difficult separations with the RediSep ${ }^{\mathrm{TM}}$ cartridge leading to extremely high values of mass of silica (Table 1 , entry 2), and partially the equations obtained with spherical silica (SNAP Ultra ${ }^{\mathrm{TM}}$, Table 1, entry 5), the values of $A$ range from 10 to 152 .

$$
m_{\mathrm{SiO}_{2}}=A m_{\mathrm{S}} \quad 10<A<152
$$

\section{Mass of eluent}

The total amount of solvent required for carrying out a chromatography is composed of the part used to pack the column, of that needed to elute the sample, (i.e., the retention volume $V_{\mathrm{R}}$ and the half width of the chromatographic peak $\omega$ (Figure 1)), and the void volume $V_{0}$ that corresponds to the mobile phase volume in the packed column.

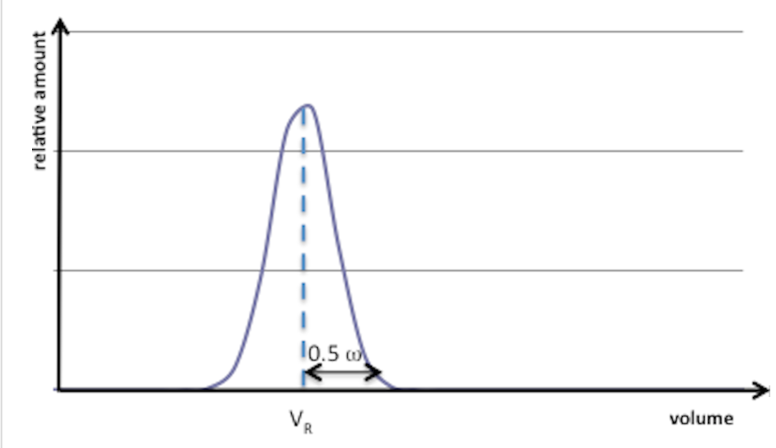

Figure 1: Chromatographic peak of a compound eluted at a retention volume $V_{R}$ with a width $\omega$.

Considering that the solvent used to pack the column is generally recycled, the volume of eluent required can then be expressed by Equation 8 .

$$
V_{\text {eluent }}=V_{\mathrm{R}}+0.5 \omega+V_{0}
$$

Under ideal conditions, the retention volume $V_{\mathrm{R}}$ can be related to the $R_{\mathrm{f}}$ by Equation 9 .

$$
V_{\mathrm{R}}^{\text {ideal }}=\frac{V_{0}}{R_{\mathrm{f}}}
$$

Some deviations of this equation were observed for silica gel column and a correction factor $\mathrm{C}$ was proposed [12], so that $V_{\mathrm{R}}$ should be calculated using Equation 10. A value of 0.64 was found for manually packed columns, while for commercial cartridges, the value of $C$ was 0.66 .

$$
V_{\mathrm{R}}=C \frac{V_{0}}{R_{\mathrm{f}}}
$$

The half width of the chromatographic peak can be estimated by assuming that the peak is described by a Gaussian with a standard deviation $\sigma$ (Equation 11).

$$
\omega=4 \sigma=4 \frac{V_{\mathrm{R}}}{\sqrt{N}}
$$

In this equation, the term $N$ represents the efficiency of the chromatographic column, i.e., the system's ability to elute the same compounds at identical rates in order to obtain thin peaks. $\mathrm{N}$ is defined as the number of theoretical plates of the column.

Using Equations 8,10 and 11, the mass of eluent can be expressed by:

$m_{\text {eluent }}=\rho_{\text {eluent }} V_{\text {eluent }}=\rho_{\text {eluent }} V_{0}\left[\frac{C}{R_{\mathrm{f}}}\left(1+\frac{2}{\sqrt{N}}\right)+1\right]$

The void volume $V_{0}$ is connected to the column volume $V_{\mathrm{C}}$ by the porosity of the silica $\varepsilon_{\mathrm{SiO}_{2}}\left(\varepsilon_{\mathrm{SiO}_{2}}=0.9\right)$ and the volume of the column depends on the mass and density $\left(\rho_{\mathrm{SiO}_{2}}=0.5\right)$ of the silica according to Equation 13 and Equation 14.

$$
\begin{gathered}
V_{0}=\varepsilon_{\mathrm{SiO}_{2}} V_{\mathrm{C}} \\
V_{C}=\frac{m_{\mathrm{SiO}_{2}}}{\rho_{\mathrm{SiO}_{2}}}
\end{gathered}
$$

We can then deduce the following equation for the mass of eluent:

$$
m_{\text {eluent }}=1.8 \rho_{\text {eluent }} m_{\mathrm{SiO}_{2}}\left[\frac{C}{R_{\mathrm{f}}}\left(1+\frac{2}{\sqrt{N}}\right)+1\right]
$$

Although $N$ depends on various parameters such as the size of the column, the packing particles, the quality of the packing and the flow of the mobile phase, an average value of 35 was proposed for flash chromatography column [16]. Alternatively, in order to take into account broadening of the chromatographic peaks due to the amount of compounds in the sample, it was proposed [12] to evaluate $N$ as a function of the mass fraction of the product in the sample $\left(m_{\mathrm{P}}=x m_{\mathrm{S}}\right)$, for difficult 
separation (Equation 16, B = 51.70) or more easier separation (Equation 16, B = 33.64).

$$
N=B(x)^{-0.44}
$$

\section{Mass intensity of a chromatography}

As already stated above, the mass intensity of purification by chromatography is the ratio between the total mass $m_{\mathrm{T}}$ used to perform the chromatography and the mass $m_{\mathrm{P}}$ of the product (Equation 6). The total mass is the sum of the silica and eluent masses that can be expressed from Equation 7 and Equation 15

$$
m_{\mathrm{T}}=A m_{\mathrm{S}}+1.8 A m_{\mathrm{S}} \rho_{\text {eluent }}\left[\frac{C}{R_{\mathrm{f}}}\left(1+\frac{2}{\sqrt{N}}\right)+1\right]
$$

Considering $\mathrm{x}$, the mass fraction of the product in the sample the theoretical expression of $M I_{\mathrm{Chr}}$ becomes:

$M I_{\mathrm{Chr}}^{\mathrm{th}}=\frac{A}{x}+\frac{1.8 A \rho_{\text {eluent }}}{x}\left[\frac{C}{R_{\mathrm{f}}}\left(1+\frac{2}{\sqrt{N}}\right)+1\right]=A^{\prime}+B^{\prime} \rho_{\text {eluent }}$

\section{Application}

We chose 4 syntheses whose crude reaction products were purified by flash chromatography to illustrate the calculations developed above (Scheme 1).
In all cases, $C$ was set at 0.64 and $M I_{\mathrm{Chr}}^{\text {th }}$ was calculated using $N_{\text {calc }}$ (Equation 16) or $N=35$. The value of $M I_{\mathrm{Chr}}^{\exp }$ was determined according to the experimental data.

Compound 1, obtained by aldol condensation (Scheme 1, reaction a) in $80 \%$ yield [8], was chromatographed on a manually packed column using as eluent a 7:3 cyclohexane-acetone mixture (Table 2, entry 1). The mass fraction of product in the crude reaction mixture (79\%) was calculated after chromatography taking into account the isolated mass of $\mathbf{1}$. The values calculated using Equation 18 with $N=35$ or $N_{\text {calc }}$ (Equation 16, $B=33.64$ or $B=51.70$ ) deviated only from 6,7 and $11 \%$ of the experimental value, respectively. Another experiment (a(bis), Table 2, entry 2) led to a crude reaction mixture containing $60 \%$ by weight of 1 which was purified using a disposable cartridge (PuriFlash SIHP $30 \mu \mathrm{m}$, Interchim) and cyclohexane-AcOEt $(7: 3)$ as the eluent. Also in this case, the calculated values were very close to the experimental ones (differences of 1,3 or $6 \%$ ).

The crude mixture of reaction $b$, a bromination in alpha position of a ketone leading to $2[17,18]$ in $54 \%$ yield, was chromatographed using AcOEt-MeOH (9:1) as the eluent [8]. The mass fraction of compound $\mathbf{2}$ in the sample was only $38 \%$, leading to high value of $M I_{\mathrm{Chr}}^{\mathrm{exp}}$ (Table 2, entry 3). The calculations lead to $M I_{\mathrm{Chr}}^{\exp }$ values having differences of 11,14 and $17 \%$ compared to the experimental value. Obviously the lower is the<smiles>C#CCOc1ccc(C(C)=O)c(O)c1</smiles><smiles>O=Cc1ccc(O)cc1</smiles><smiles>C#CCOc1ccc(C(=O)/C=C/c2ccc(O)cc2)c(O)c1</smiles><smiles>CC(=O)CC1OC(O)C(O)C(O)C1O</smiles><smiles>O=C(CBr)CC1OC(O)C(O)C(O)C(O)C1O</smiles><smiles>OCC(CO)(CO)CO</smiles><smiles>Br[13CH2]Br</smiles><smiles>C1CCCC1</smiles>

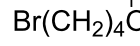

3

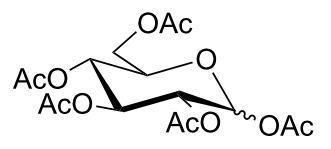
EtSH

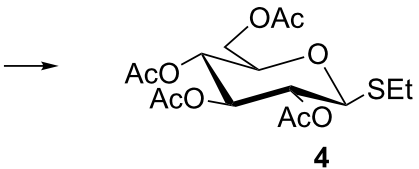


Table 2: Comparison of the experimental values of the mass intensity of chromatography $\left(M I_{\mathrm{Chr}}^{\exp }\right)$ with the theoretical estimated values ( $\left.M I_{\mathrm{Chr}}^{\text {th }}\right)$ for various reactions (Scheme 1).

\begin{tabular}{|c|c|c|c|c|c|c|c|c|c|c|}
\hline Entry & Reaction & $R_{\mathrm{f}}$ & $A$ & $x$ & $A^{\prime}$ & $\rho_{\text {eluent }}$ & $N$ & $B^{\prime}$ & $M I_{\mathrm{Chr}}^{\mathrm{th}^{\mathrm{a}}}$ & $M I_{\mathrm{Chr}}^{\exp }$ \\
\hline 1 & a & 0.1 & 49 & 0.79 & 62 & 0.78 & $\begin{array}{c}35 \\
37^{b} \\
57^{c}\end{array}$ & $\begin{array}{l}1072 \\
1065 \\
1019\end{array}$ & $\begin{array}{l}901 \\
896 \\
860\end{array}$ & 962 \\
\hline 2 & $a(b i s)^{d}$ & 0.15 & 47 & 0.60 & 78 & 0.81 & $\begin{array}{l}35 \\
42^{b} \\
65^{c}\end{array}$ & $\begin{array}{l}946 \\
928 \\
892\end{array}$ & $\begin{array}{l}843 \\
829 \\
800\end{array}$ & 857 \\
\hline 3 & $b$ & 0.13 & 30 & 0.38 & 79 & 0.89 & $\begin{array}{c}35 \\
51^{b} \\
79^{c}\end{array}$ & $\begin{array}{c}1074 \\
1033 \\
995\end{array}$ & $\begin{array}{c}1031 \\
994 \\
961\end{array}$ & 1161 \\
\hline 4 & $\mathrm{C}$ & 0.30 & 20 & 0.42 & 47 & 0.65 & $\begin{array}{c}35 \\
49^{b} \\
76^{c}\end{array}$ & $\begin{array}{l}324 \\
314 \\
304\end{array}$ & $\begin{array}{l}258 \\
252 \\
245\end{array}$ & 250 \\
\hline 5 & $d$ & 0.20 & 30 & 0.61 & 49 & 0.81 & $\begin{array}{l}35 \\
42^{b} \\
64^{c}\end{array}$ & $\begin{array}{l}468 \\
459 \\
442\end{array}$ & $\begin{array}{l}427 \\
421 \\
407\end{array}$ & 458 \\
\hline
\end{tabular}


Equation 16 with $B=51.70$. ${ }^{\text {Reaction }}$ a, other experimental conditions.

proportion by weight of the compound in the sample, the higher is the mass intensity for the chromatography. This variation in $(1 / x)$ was represented for reaction $b$ in Figure 2. Therefore when this proportion is not precisely known, which is the most frequent case before performing the purification, it is possible to estimate a minimum value of $M I_{\mathrm{Chr}}$ setting $x=1$, or, if the mass of the sample to be purified is higher than the theoretical mass of product, $x$ can be calculated assuming a $100 \%$ yield (Equation 19).

$$
x_{\max }=\frac{M_{\mathrm{W}} \text { (product)*number of mole of substrate }}{m_{\mathrm{s}}}
$$

It is also clear that if a treatment (e.g. extraction) can reduce the mass of the sample to be purified, it would reduce the mass intensity related to chromatography. This should obviously not be to the detriment of the overall mass balance.

This can be illustrated by example c (Scheme 1). In fact, this alkylation reaction was carried out in the presence of a large excess (4 equiv) of dibromobutane to get compound $\mathbf{3}$ in a good yield (73\%) [19]. Some of this excess was removed from the crude reaction product by distillation, reducing the mass of the sample by $53 \%$. This allowed to recycle the reactant but also to greatly reduce the weight of silica to be used $(A=20)$ and, accordingly the mass of solvent (Table 2, entry 4 ). This purification with a particularly low $M I_{\mathrm{Chr}}$, compared to the other examples, corresponded to a filtration on silica gel rather than to a flash chromatography.

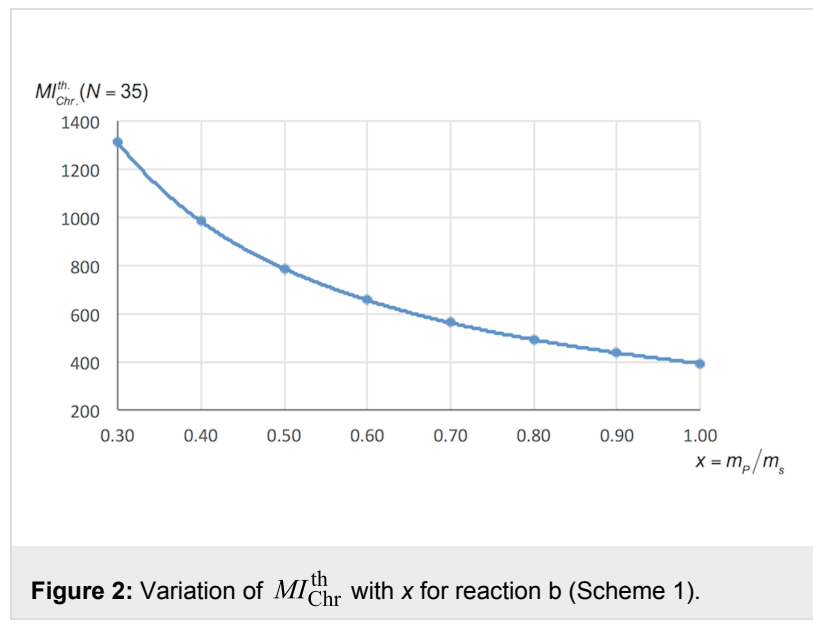

The last example (Scheme 1d) is a $S$-glycosylation (isolated yield $=62 \%$ ) leading to compound 4 [20]. For this crude reaction mixture containing $61 \%$ of 4 , a correct separation was obtained on TLC with the mobile phase cyclohexane-EtOAc (75:25). Again, the values obtained by the calculation were close to the experimental ones, with differentials of 7,8 and $11 \%$ depending on the value taken for $N$ (Table 2 , entry 5 ).

In each case, the calculation afforded values close (deviations $<17 \%$ ) to the experimental value (Figure 3). As already pointed out above, the calculation depends on the value of $x$ that it is not always easy to estimate, but it is possible to estimate a minimum of the mass of intensity related to the chromatography by setting $x$ closed to 1 . 


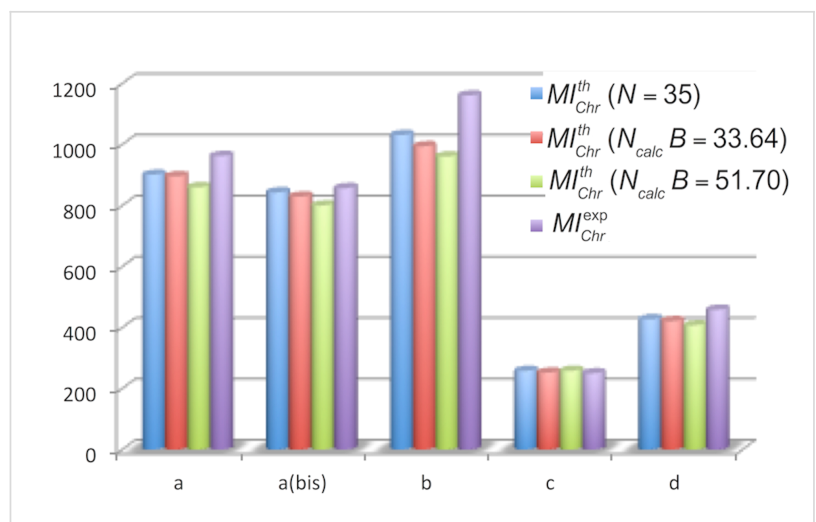

Figure 3: Comparison between calculated and experimental values of $M I_{\mathrm{Chr}}$ for the reactions of Scheme 1.

The value of $M I_{\mathrm{Chr}}$ also depends on the retention factor $\left(R_{\mathrm{f}}\right)$, especially when the latter is less than 0.2 (Figure 4). An estimation of the minimum is also possible by setting an $R_{\mathrm{f}}$ to a value close to 0.35 , as recommended in the seminal paper of Still et al. [10].

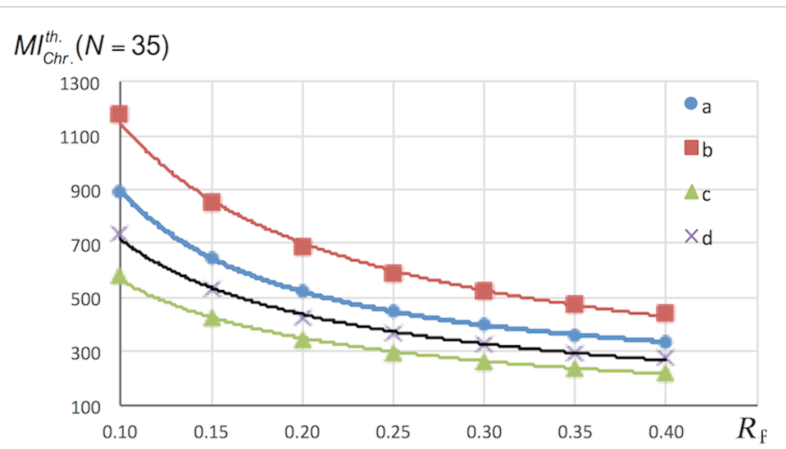

Figure 4: Variation of $M I_{\mathrm{Chr}}^{\mathrm{th}}(N=35)$ with $R_{\mathrm{f}}$ for the reactions of Scheme 1.

\section{Conclusion}

If the impact of chromatography on the environmental factor $E$ of a process seems pretty obvious, we have developed here a tool to quantify it. In an extremely favourable case with a $95 \%$ pure sample $(x=0.95)$, a very easy separation achievable with a small mass of silica $(\mathrm{A}=10)$ and $R_{\mathrm{f}}=0.35$, we find, for low density eluent (0.6), an $M I_{\mathrm{Chr}}$ value close to 50 . By doubling the amount of silica, which is closer to reality, the $M I_{\mathrm{Chr}}$ value is about 100 . In real cases chosen here as examples, we have shown that the values were fairly between about 200 and 1200 .

Since it is clear that chromatography should be avoided wherever possible, works proposing alternative purification methods have been published $[9,21,22]$. When the purification by flash chromatography is necessary, solvents with low environmental impacts should be used [23-25]. In this context, super critical chromatography which allows to obtain very low retention volumes and easy recycling offers an interesting alternative [26] but requires a significant investment.

\section{Acknowledgements}

We thank the Ministère de l'Enseignement Supérieur et de la Recherche, the Centre National de la Recherche Scientifique and the Université Paris-Sud for financial support.

\section{References}

1. Sheldon, R. A. CHEMTECH 1994, 24, 38-47.

2. Sheldon, R. A. Green Chem. 2007, 9, 1273-1283. doi:10.1039/b713736m

3. Curzons, A. D.; Constable, D. J. C.; Mortimer, D. N.; Cunningham, V. L. Green Chem. 2001, 3, 1-6. doi:10.1039/b007871i

4. Constable, D. J. C.; Curzons, A. D.; Cunningham, V. L. Green Chem 2002, 4, 521-527. doi:10.1039/B206169B

5. Eissen, M.; Metzger, J. O. Chem. - Eur. J. 2002, 8, 3580-3585. doi:10.1002/1521-3765(20020816)8:16<3580::AID-CHEM3580>3.0.C O;2-J

6. Augé, J. Green Chem. 2008, 10, 225-231. doi:10.1039/B711274B

7. Augé, J.; Scherrmann, M.-C. New J. Chem. 2012, 36, 1091-1098. doi:10.1039/c2nj20998e

8. Pessel, F.; Billault, I.; Scherrmann, M.-C. Green Chem. 2016, 18, 5558-5568. doi:10.1039/C6GC01647B

9. Peterson, E. A.; Dillon, B.; Raheem, I.; Richardson, P.; Richter, D.; Schmidt, R.; Sneddon, H. F. Green Chem. 2014, 16, 4060-4075. doi:10.1039/C4GC00615A

10. Still, W. C.; Kahn, M.; Mitra, A. J. Org. Chem. 1978, 43, 2923-2925. doi:10.1021/jo00408a041

11. Stevens, W. C., Jr.; Hill, D. C. Mol. Diversity 2009, 13, 247-252. doi:10.1007/s11030-008-9104-x

12. Fair, J. D.; Kormos, C. M. J. Chromatogr. A 2008, 1211, 49-54. doi:10.1016/j.chroma.2008.09.085

13. Effective Organic Compound Purification - Guideline and tactics for Flash Chromatography, 4th ed.; Teledyne Isco Inc, 2010. (www.isco.com).

14. New Generation of Ready to Connect Cartridges using MERCK silica. EasyVarioFlash $®$ (Götec-Labortechnik GmbH), 2007; http://www.swisslabs.eu/uploads/files/Flash_Chromatography_New_G eneration_ReadytoUse_Cartridges.pdf.

15. Biotage Flash Cartridge User Guide - The Definitive Guide to Flash Chromatography. Biotage, 2014; http://www.biotage.com/product-group/flash-cartridges.

16. Meyer, V. R. Practical high-performance liquid chromatography; Wiley, 2010. doi:10.1002/9780470688427

17. Howard, S.; Withers, S. G. J. Am. Chem. Soc. 1998, 120, 10326-10331. doi:10.1021/ja981580r

18. Billault, I.; Pessel, F.; Petit, A.; Turgis, R.; Scherrmann, M.-C. New J. Chem. 2015, 39, 1986-1995. doi:10.1039/C4NJ01784F

19. Turgis, R.; Billault, I.; Acherar, S.; Augé, J.; Scherrmann, M.-C. Green Chem. 2013, 15, 1016-1029. doi:10.1039/c3gc37097f

20. Ferrier, R. J.; Furneaux, R. H. Carbohydr. Res. 1976, 52, 63-68. doi:10.1016/S0008-6215(00)85946-7

21. Prosa, N.; Turgis, R.; Piccardi, R.; Scherrmann, M.-C. Eur. J. Org. Chem. 2012, 2188-2200. doi:10.1002/ejoc.201101726

22. Weiß, M.; Brinkmann, T.; Gröger, H. Green Chem. 2010, 12, 1580-1588. doi:10.1039/c002721a 
23. MacMillan, D. S.; Murray, J.; Sneddon, H. F.; Jamieson, C.; Watson, A. J. B. Green Chem. 2012, 14, 3016-3019. doi:10.1039/c2gc36378j

24. Taygerly, J. P.; Miller, L. M.; Yee, A.; Peterson, E. A. Green Chem. 2012, 14, 3020-3025. doi:10.1039/c2gc36064k

25. Pena-Pereira, F.; Kloskowski, A.; Namiesnik, J. Green Chem. 2015, 17, 3667-3705. doi:10.1039/C5GC00611B

26. Miller, L.; Mahoney, M. J. Chromatogr. A 2012, 1250, 264-273. doi:10.1016/j.chroma.2012.06.029

\section{License and Terms}

This is an Open Access article under the terms of the Creative Commons Attribution License

(http://creativecommons.org/licenses/by/4.0), which permits unrestricted use, distribution, and reproduction in any medium, provided the original work is properly cited.

The license is subject to the Beilstein Journal of Organic Chemistry terms and conditions:

(http://www.beilstein-journals.org/bjoc)

The definitive version of this article is the electronic one which can be found at: $\underline{\text { doi: } 10.3762 / \text { bjoc. } 12.228}$ 